\title{
ON ARRANGEMENTS OF PLANE REAL QUINTICS WITH RESPECT TO A PAIR OF LINES
}

\author{
A. B. KORCHAGIN AND G. M. POLOTOVSKII
}

\begin{abstract}
The arrangements of an $M$-curve of degree 5 with respect to a pair of lines in the projective plane are studied under the natural conditions of general position and maximality.
\end{abstract}

\section{§1. Problem setting and formulation of the Result}

In this paper we continue the classification started in [1]. As a model of the projective plane $\mathbb{R} P^{2}$, we use the Poincaré disk. A real projective plane curve $C_{n}$ of degree $n$ is a real homogeneous polynomial in three variables $x, y, z$ defined up to a nonzero constant factor. The set of zeros of $C_{n}$ in the real (complex) projective plane $\mathbb{R} P^{2}$ (respectively, $\mathbb{C} P^{2}$ ) is called the set of real (respectively, complex) points of $C_{n}$ and is denoted by $\mathbb{R} C_{n}$ (respectively, $\mathbb{C} C_{n}$ ).

Let $C_{1}$ and $C_{1}^{\prime}$ be two distinct lines. The set $\mathbb{R} P^{2} \backslash\left(\mathbb{R} C_{1} \cup \mathbb{R} C_{1}^{\prime}\right)$ consists of two connected components, each homeomorphic to an open disk. We denote by $D_{1}$ and $D_{2}$ the closures of these components. Our aim is to find a topological classification of the quadruples

$$
\left(\mathbb{R} P^{2}, \mathbb{R} C_{5} \cup \mathbb{R} C_{1} \cup \mathbb{R} C_{1}^{\prime}, \mathbb{R} C_{5} \cup \mathbb{R} C_{1}, \mathbb{R} C_{1}\right)
$$

under the following conditions of maximality and general position.

(i) The quintic curve $C_{5}$ is an $M$-curve, which means that the set $\mathbb{R} C_{5}$ of its real points consists of the odd branch $J$ and six ovals that do not surround one another.

(ii) The curves $\mathbb{R} C_{5}, \mathbb{R} C_{1}, \mathbb{R} C_{1}^{\prime}$ are in general position, i.e., they have pairwise transversal intersection, and the curve $\mathbb{R} C_{5}$ does not pass through the point of intersection of the lines $\mathbb{R} C_{1}$ and $\mathbb{R} C_{1}^{\prime}$.

(iii) Each of the lines $\mathbb{R} C_{1}$ and $\mathbb{R} C_{1}^{\prime}$ intersects the odd branch $J$ of the curve $\mathbb{R} C_{5}$ at five points.

(iv) Let $a$ and $b$ be the numbers of the arcs of $J$ that lie in the disks $D_{1}$ and $D_{2}$ (respectively) and have one endpoint lying on the line $\mathbb{R} C_{1}$ and the other on $\mathbb{R} C_{1}^{\prime}$. In this paper we consider the case where $(a, b)=(1,3)$.

Remark. By the equivalence of the disks $D_{1}$ and $D_{2}$, we may assume that $(a, b) \in$ $\{(1,1),(1,3),(1,5),(3,3),(3,5)\}$. Classification for $(a, b)=(1,1)$ was found in [1]. For $(1,5)$ and $(3,5)$ classification follows easily from that of the arrangements of a quintic curve with respect to a line; see 2. For $(3,3)$, classification was obtained recently by S. Yu. Orevkov in 3.

The following theorem gives the answer for $(a, b)=(1,3)$.

2000 Mathematics Subject Classification. Primary 14H50.

Key words and phrases. Plane real algebraic curve, splitting algebraic curve, topological classification. 
Theorem 1. The union of a quintic curve and a pair of lines is realized by exactly 51 distinct topological models of quadruples (1.1) satisfying condition (i)-(iv); the numbers of the codes of the realizable models are shown in bold in Table 1 below.

Remark. The proof of this theorem was completed by S. Yu. Orevkov in [3]; see $\S 5$ below for the details. Pictures of realizable models are listed in Subsection 0.7 of [3; we do not reproduce them in our paper.

To prove Theorem 1, we use the same method that was applied to prove a similar theorem in [1: first, we list all topological models of the quadruple (1.1) satisfying conditions (i)-(iv) that do not contradict the Bézout theorem and complex orientation formulas; second, to prove the nonrealizability of some models by algebraic curves, we use the Orevkov method based on the link theory (see [4]). After that, we construct algebraic curves by the Viro patchworking method (see [5, 6, 7]). As in [1, the enumeration of the admissible arrangements and the nonrealizability of models was proved by the second author, and the construction of algebraic models was proved by the first author.

We thank S. Yu. Orevkov for useful discussions.

\section{§2. EnUmeration of ADMissible topological MODEL}

For uniformity of our pictures, we place the point $\mathbb{R} C_{1} \cap \mathbb{R} C_{1}^{\prime}$ at the ends of the horizontal diameter of the Poincare disk, we select the disk $D_{2}$ so that it intersects the boundary of the Poincaré disk only at the point $\mathbb{R} C_{1} \cap \mathbb{R} C_{1}^{\prime}$, and we assume that the upper line in the pictures is $\mathbb{R} C_{1}$. The quadruples (1.1) will be coded as follows.

1) The points of intersection of the odd branch $J$ with the line $\mathbb{R} C_{1}$ (respectively, $\mathbb{R} C_{1}^{\prime}$ ) are enumerated from left to right along this line by the symbols $0,1,2,3,4$ (respectively, from right to left by the symbols $5,6,7,8,9)$.

2) The location of $J$ relative to $\mathbb{R} C_{1} \cup \mathbb{R} C_{1}^{\prime}$ is coded by a permutation $\left(i_{1}, i_{2}, \ldots, i_{10}\right)$ of the symbols $0,1, \ldots, 9$. This permutation shows the order in which the odd branch $J$ passes through the points $0,1, \ldots, 9$. Here we assume that only the arc $\left(i_{1}, i_{10}\right)$ of $J$ that contains no points of the lines $\mathbb{R} C_{1}$ and $\mathbb{R} C_{1}^{\prime}$ different from $i_{1}$ and $i_{10}$ intersects the boundary of the Poincaré disk.

3) Permutations are equipped with additional symbols in angle brackets or in braces: a fragment $i_{s}\langle m\rangle i_{s+1}$ (respectively, $i_{s}\{m\} i_{s+1}$ ) means that if one moves along the arc $\left(i_{s}, i_{s+1}\right)$ from $i_{s}$ to $i_{s+1}$, then the region on the right (respectively, on the left) contains $m$ ovals of the curve $C_{5}$.

Remark. Clearly, for every model of the curve $\mathbb{R} C_{5} \cup \mathbb{R} C_{1} \cup \mathbb{R} C_{1}^{\prime}$ in $\mathbb{R} P^{2}$, its code defined by the rules 1)-3) is not unique. We shall always choose a permutation minimal in the lexicographical order and write the additional symbols showing the location of ovals arbitrarily in a certain possible way.

First, under the conditions (ii)-(iv), we list all possible locations of the odd branch $J$ with respect to $\mathbb{R} C_{1} \cup \mathbb{R} C_{1}^{\prime}$. By simple exhaustive search, we find that, up to symmetry, three arcs of $J$ in the disk $D_{2}$ can connect a triple of points on $\mathbb{R} C_{1}$ with a triple of points on $\mathbb{R} C_{1}^{\prime}$ only in six different ways depicted in Figure1. After that, in each of these cases it is necessary to complete the odd branch $J$ in all possible ways so that condition (iv) will be fulfilled. For example, for the first case in Figure 1 it is easy to get the two arrangements shown in Figure 2,

There are exactly 20 pairwise different arrangements for $\mathbb{R} C_{1} \cup \mathbb{R} C_{1}^{\prime} \cup J$ that can be obtained from the six cases depicted in Figure 1. For each of these 20 arrangements the set $\mathbb{R} P^{2} \backslash\left(\mathbb{R} C_{1} \cup \mathbb{R} C_{1}^{\prime} \cup J\right)$ consists of 12 connected components (homeomorphic to an open disk). The next step is to find all admissible arrangements of the six ovals of a quintic curve in these 12 regions. 

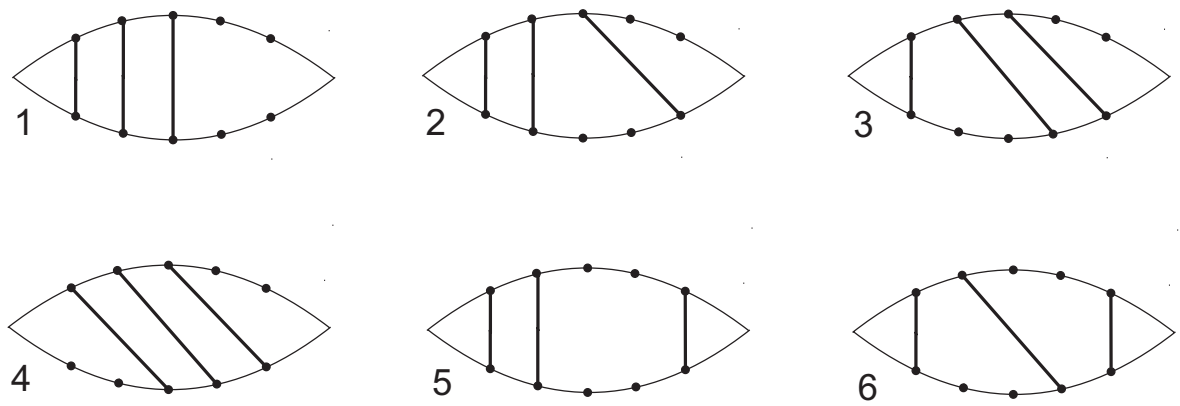

FiguRE 1

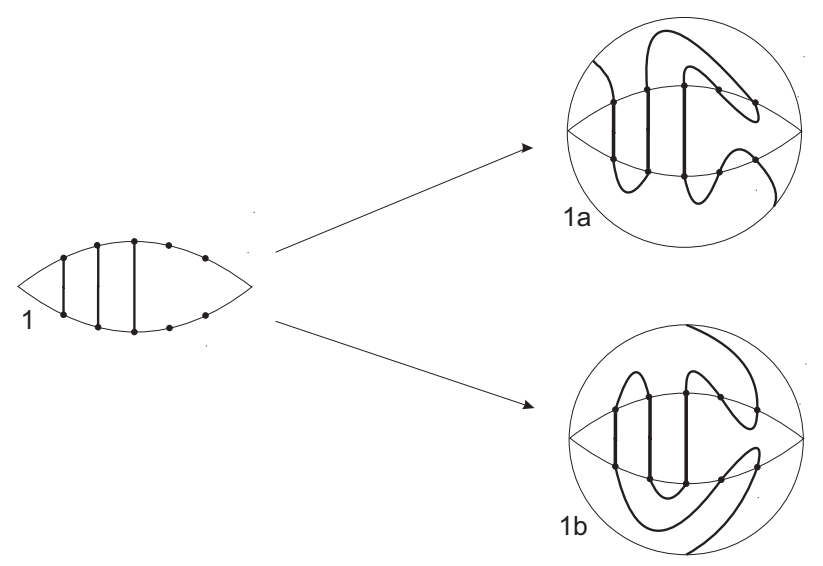

FiguRE 2

It turns out that, as in [1, a substantial part of the admissible arrangements of ovals is ruled out by the Bézout theorem, which we apply in two ways. First, if for a given model $\mathbb{R} C_{1} \cup \mathbb{R} C_{1}^{\prime} \cup J$ one can produce a pencil of lines that covers some region and consists of lines, each having 7 points of intersection with $\mathbb{R} C_{1} \cup \mathbb{R} C_{1}^{\prime} \cup J$, then this region can contain no ovals of our quintic curve. Second, if for a conjectural arrangement of ovals one smooths in some way the double points of $\mathbb{R} C_{1} \cup \mathbb{R} C_{1}^{\prime} \cup J$, obtaining a number of nests with total number of weights exceeding 1 , then such an arrangement of ovals is impossible. In particular, for the model with the code (0789614325), the second method proves that no oval can be put in any region. Thus, only 19 cases out of the 20 mentioned in the preceding paragraph remain.

After an application of the Bézout theorem (in the same manner as in [1]), some of the remaining cases contradict the classification of the unions of a line and a quintic curve as presented in [2]. Namely, for the model $\mathbb{R} C_{1} \cup \mathbb{R} C_{1}^{\prime} \cup J$ depicted in Figure 3, the Bézout theorem allows the location of ovals only in regions marked by Latin letters. However, in 2 it was proved that any topological pair $\left(\mathbb{R} P^{2}, \mathbb{R} C_{1} \cup \mathbb{R} C_{5}\right)$ must be homeomorphic to one of the two topological pairs depicted in the right upper corner of Figure 3, If we denote the number of ovals in a given region by the same Latin letter, then we obtain the following disjunction of linear systems:

$$
\left\{\begin{array}{l}
E=2, \\
B+D=4
\end{array} \bigvee E=6 \bigvee\left\{\begin{array}{l}
A+C+F=2, \\
B+D=4
\end{array} \quad \bigvee A+C+F=6\right.\right.
$$


In [2] it was also proved that a topological pair $\left(\mathbb{R} P^{2}, \mathbb{R} C_{1}^{\prime} \cup \mathbb{R} C_{5}\right)$ must be homeomorphic to one of the two topological pairs depicted in the right lower corner of Figure 3 , whence it follows that

$$
\begin{gathered}
\left\{\begin{array}{l}
D+E=1, \\
B+C=5
\end{array}\right. \\
\bigvee\left\{\begin{array} { l } 
{ D + E = 5 , } \\
{ B + C = 1 }
\end{array} \bigvee \left\{\begin{array} { l } 
{ F = 1 , } \\
{ B + C = 5 } \\
{ B + C = 5 }
\end{array} \bigvee \left\{\begin{array}{l}
A=1, \\
B+C=1 .
\end{array}\right.\right.\right.
\end{gathered}
$$

For this example, the nonnegative integer solutions of (2.1) and (2.2) provide the set of

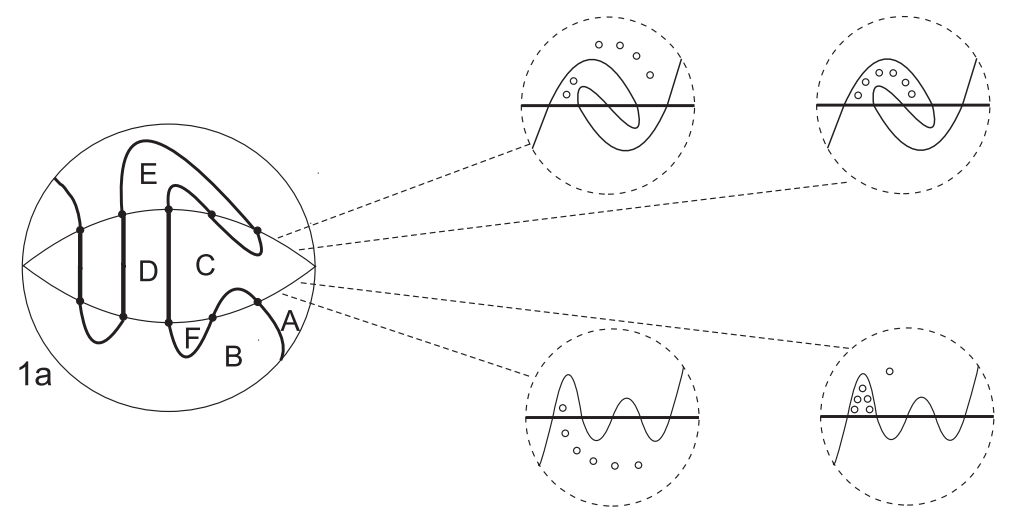

FigurE 3

admissible models, which are subject to subsequent steps of the study (see rows 6-13 in Table 1).

We complete the enumeration of the admissible topological models in precisely the same way as in [1. We fix the orientation of the lines $\mathbb{R} C_{1}, \mathbb{R} C_{1}^{\prime}$ and of the odd branch $J$; we smooth all double points of $\mathbb{R} C_{5} \cup \mathbb{R} C_{1} \cup \mathbb{R} C_{1}^{\prime}$ in accordance with the fixed orientations and get a nonsingular curve of degree 7. Applying the complex orientation formulas to $\mathbb{R} C_{5}$ and to the curve of degree 7 obtained, we see that the nine models with the codes $(012\{5\} 98\langle 1\rangle 34765),(012\{a\} 9876\langle 6-a\rangle 345),(0\langle a\rangle 12\{6-a\} 9876345),(0\langle a\rangle 98\{6-$ $a\} 7612345)$, where $a \in\{1,5\}$, and $(09\langle 5\rangle 87\{1\} 612345)$ contradict these formulas.

The list of codes of the remaining topological models (1.1), which are subject to further examination, is shown in the third column of Table 1.

\section{§3. RESTRICTION BY LINK THEORY METHODS}

The Orevkov method of finding restrictions on isotopy types of an algebraic curve is presented in the literature in detail. Besides the fundamental paper [4, see also [1, 8, 9]. Since an application of this method in our paper is absolutely the same as in [1], we confine ourselves to a short description and an example.

Suppose that a curve $C_{7}$ has only double points and all of them are generic. Suppose that a pencil $L_{P}$ with center at $P \in \mathbb{R} P^{2} \backslash \mathbb{R} C_{7}$ is in maximal general position relative to $\mathbb{R} C_{7}$, which means that (i) every line $l \in L_{P}$ intersects the curve $\mathbb{R} C_{7}$ in at least five distinct points, (ii) there is a line in $L_{P}$ that intersects $\mathbb{R} C_{7}$ at seven points, and (iii) every line $l \in L_{P}$ contains at most one double point of the curve $\mathbb{R} C_{7}$ or at most one point of tangency with it. Consider the set $M=\mathbb{C} C_{7} \cap \mathbb{C} L_{P} \subset \mathbb{C} P^{2}$, where $\mathbb{C} L_{P}$ is the pencil of complex lines with center at $P$. The set $M$ is homeomorphic to a collection of circles glued pairwise at the double points of the curve $\mathbb{R} C_{7}$ and at the points of tangency of the 
TABle 1

\begin{tabular}{|c|c|c|c|c|}
\hline No. & $\begin{array}{c}\mathbb{R} C_{1} \cup \mathbb{R} C_{1}^{\prime} \cup J \text { and } \\
\text { choice of pencil center }\end{array}$ & $\begin{array}{l}\mathbb{R} C_{1} \cup \mathbb{R} C_{1}^{\prime} \cup \mathbb{R} C_{5} \\
\text { (location of ovals) }\end{array}$ & $h$ & Answer \\
\hline $\mathbf{1}$ & $(0127\{P\} 634589)$ & $(0\langle 3\rangle 1\{2\} 276345\{1\} 89)$ & 0 & $8.1 \uplus_{1} 9.9^{*}$ \\
\hline 2 & $-11-$ & $(01\{5\} 2\{1\} 7634589)$ & 0 & $8.1 \uplus_{1} 9.9$ \\
\hline 3 & $-11-$ & $(01\{1\} 2\{5\} 7634589)$ & $>0$ & \\
\hline 4 & $\overline{-11-}$ & $(0\langle 3\rangle 127\langle 2\rangle 6345\{1\} 89)$ & $>0$ & \\
\hline 5 & $-11-$ & $(0\langle 4\rangle 1\{1\} 2\{1\} 7634589)$ & $>0$ & \\
\hline 6 & $(0127\langle P\rangle 896345)$ & 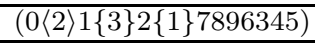 & 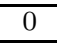 & $8.2 \uplus_{1} 9.3^{*}$ \\
\hline 7 & $-11-$ & $(0\langle 5\rangle 1\langle 1\rangle 27896345)$ & 0 & $13 \uplus_{3} 15.2$ \\
\hline 8 & $-11-$ & $(01\{1\} 2\{3\} 7\{2\} 896345)$ & 0 & $8.2 \uplus_{1} 9.3$ \\
\hline 9 & $\overline{-11-}$ & $(0\langle 1\rangle 1\langle 1\rangle 2789\langle 4\rangle 6345)$ & $>0$ & \\
\hline 10 & $\overline{-11-}$ & $(0\langle 1\rangle 127896345\{5\})$ & $>0$ & \\
\hline 11 & $\overline{-11-}$ & $(0\langle 1\rangle 1\langle 5\rangle 27896345)$ & $>0$ & \\
\hline 12 & $-11-$ & $(0\langle 5\rangle 127896345\{1\})$ & $>0$ & \\
\hline 13 & $-11-$ & $(0\langle 1\rangle 1\{4\} 27896345\{1\})$ & $>0$ & \\
\hline 14 & $(012967\langle P\rangle 8345)$ & $\overline{(012\{1\} 9\langle 3\rangle 678\langle 2\rangle 345)}$ & 0 & $8.2 \uplus_{1} 9.4^{*}$ \\
\hline 15 & $-11-$ & $(0129\{2\} 678\{3\} 3\{1\} 45)$ & 0 & $8.2 \uplus_{1} 9.4$ \\
\hline 16 & $-11-$ & $(0129678\langle 5\rangle 3\langle 1\rangle 45)$ & 0 & $\begin{array}{lllll}\text { restricted in }[3 & 4.1] & \mathrm{PH}\end{array}$ \\
\hline 17 & 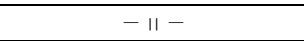 & $(0129678\langle 1\rangle 3\langle 5\rangle 45)$ & $>0$ & \\
\hline 18 & $-11-$ & $(0129\langle 4\rangle 8\langle 1\rangle 3\langle 1\rangle 4567)$ & $>0$ & \\
\hline 19 & $(012983456\{P\} 7)$ & $(0\langle 2\rangle 12\{1\} 9\langle 3\rangle 834567)$ & 0 & $8.1 \uplus_{1} 9.6^{*}$ \\
\hline 20 & $-11-$ & $(0\langle 5\rangle 1298345\{1\} 67)$ & 0 & $8.1 \uplus_{1} 9.6$ \\
\hline 21 & $\begin{array}{ll}-11- \\
-1\end{array}$ & $(0129\langle 4\rangle 8\langle 1\rangle 3\langle 1\rangle 4567)$ & 0 & constructed in [3, 2.3] \\
\hline 22 & 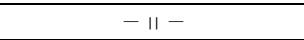 & $(01298\langle 1\rangle 3\langle 5\rangle 4567)$ & 0 & constructed in [3, 2.3] \\
\hline 23 & $-11-$ & $(01298\langle 5\rangle 3\langle 1\rangle 4567)$ & $>0$ & \\
\hline 24 & $-11-$ & $(0\langle 1\rangle 129\langle 4\rangle 8345\{1\} 67)$ & $>0$ & \\
\hline 25 & $-11-$ & $(0\langle 1\rangle 1298345\{5\} 67)$ & $>0$ & \\
\hline 26 & $-11-$ & $(012\{1\} 9\langle 3\rangle>8\langle 2\rangle 34567)$ & $>0$ & \\
\hline$\overline{\overline{27}}$ & 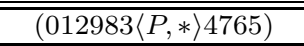 & $(01\{1\} 2983\langle 5\rangle 4765)$ & 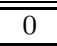 & constructed in [3, 2.3$]$ \\
\hline 28 & $-11-$ & $(01\{5\} 2983\langle 1\rangle 4765)$ & 0 & constructed in [3, 2.3$]$ \\
\hline$\overline{\overline{29}}$ & $\overline{(012\{P, *\} 9876345)}$ & 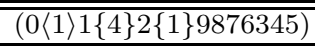 & 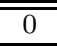 & $8.8 .1 \uplus_{1} 9.3^{*}$ \\
\hline 30 & $-11-$ & $(01\{1\} 298\{4\} 7\{1\} 6345)$ & 0 & $8.1 \uplus_{1} 9.3$ \\
\hline 31 & $\overline{-11-}$ & $(01\{1\} 29\{1\} 876\{4\} 345)$ & 0 & $8.1 \uplus_{1} 9.4$ \\
\hline 32 & $-11-$ & $(01\{4\} 2\{1\} 9876\langle 1\rangle 345)$ & 0 & $8.1 \uplus_{1} 9.4^{*}$ \\
\hline 33 & $\overline{-11-}$ & $(01298\{1\} 76\langle 5\rangle 345)$ & $>0$ & \\
\hline 34 & $-11-$ & $(012\langle 5\rangle 9876\{1\} 345)$ & $>0$ & \\
\hline 35 & $-11-$ & $(01298\{5\} 76\langle 1\rangle 345)$ & $>0$ & \\
\hline 36 & $-11-$ & $(012\langle 1\rangle 9876\{5\} 345)$ & $>0$ & \\
\hline 37 & $(01\langle P, *\rangle 29876345)$ & $(01\langle 1\rangle 2\langle 4\rangle 987\langle 1\rangle 6345)$ & 0 & $13 \uplus_{3} 15.1$ \\
\hline 38 & $-11-$ & $(01\langle 5\rangle 2987\langle 1\rangle 345)$ & $>0$ & \\
\hline 39 & $-11-$ & $(01\langle 1\rangle 298763\{5\} 45)$ & $>0$ & \\
\hline 40 & $(01298763\langle P, *\rangle 45)$ & $(01\{1\} 298763\langle 5\rangle 45)$ & $>0$ & \\
\hline 41 & $-11-$ & $(01\{1\} 29876\langle 4\rangle 3\langle 1\rangle 45)$ & 0 & $\begin{array}{llll}\text { restricted in }\left[\begin{array}{ll}3 & 0.1\end{array}\right] \mathrm{PH} \\
\end{array}$ \\
\hline 42 & $-11-$ & $(01\{5\} 298763\langle 1\rangle 45)$ & $>0$ & \\
\hline 43 & $(0129\{P, *\} 876345)$ & $(01\{1\} 29\{5\} 876345)$ & $>0$ & \\
\hline 44 & $(0129\{P, *\} 876345)$ & $(0129\{1\} 8763\{5\} 45)$ & $>0$ & \\
\hline 45 & $(012987\{P, *\} 6345)$ & $(0129\langle 1\rangle 87\{5\} 6345)$ & $>0$ & \\
\hline 46 & $-11-$ & $(0129\langle 5\rangle 87\{1\} 6345)$ & $>0$ & \\
\hline 47 & $(0\langle P, *\rangle 129876345)$ & $(0\langle 4\rangle 129\langle 1\rangle 876345\{1\})$ & 0 & $8.1 \uplus_{1} 9.1$ \\
\hline 48 & $\begin{array}{c}(0129876345) \\
\text { (no necessary pencil) }\end{array}$ & $(012987\langle 1\rangle 6345\{5\})$ & - & $\begin{array}{l}\text { restricted by reduction } \\
\text { to } C_{2} \cup C_{5} \text { in }[3\end{array}$ \\
\hline 49 & $-11-$ & $(01\{5\} 29876345\{1\})$ & - & $-\|-$ \\
\hline
\end{tabular}




\begin{tabular}{|c|c|c|c|c|}
\hline No. & $\begin{array}{l}\mathbb{R} C_{1} \cup \mathbb{R} C_{1}^{\prime} \cup J \text { and } \\
\text { choice of pencil center }\end{array}$ & $\begin{array}{l}\mathbb{R} C_{1} \cup \mathbb{R} C_{1}^{\prime} \cup \mathbb{R} C_{5} \\
\text { (location of ovals) }\end{array}$ & $\bar{h}$ & Answer \\
\hline 50 & $(014563\{P\} 2987)$ & $(0145\{4\} 63298\{2\} 7)$ & 0 & $8.2 \uplus_{1} 8.9$ \\
\hline 51 & $-11-$ & $(0145\{2\} 632\langle 2\rangle 9\langle 2\rangle 87)$ & 0 & $8.2 \uplus_{1} 9.9^{*}$ \\
\hline 52 & $-11-$ & $(01\langle 2\rangle 45632\langle 2\rangle 9\langle 2\rangle 87)$ & $>0$ & \\
\hline 53 & $-11-$ & $(0\langle 4\rangle 145632\{2\} 987)$ & $>0$ & \\
\hline 54 & $-11-$ & $(014563298\{6\} 7)$ & $>0$ & \\
\hline$\overline{55}$ & $(01456783\{P\} 29)$ & $(0145678\{5\} 329\{1\})$ & 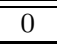 & $13 \uplus_{3} 15.3$ \\
\hline 56 & $-11-$ & $(0\langle 4\rangle 145678\{1\} 329\{1\})$ & 0 & constructed in $[3,2.3]$ \\
\hline 57 & $-11-$ & $(0\langle 3\rangle 1\langle 2\rangle 45678329\langle 1\rangle)$ & 0 & \begin{tabular}{ll|l} 
restricted in $\left[\begin{array}{lll}3 & 4.2\end{array}\right] \mathrm{PH}$ \\
\end{tabular} \\
\hline 58 & $\begin{array}{ll}-\|- \\
-11\end{array}$ & $(0\langle 3\rangle 1\{1\} 45678329\{2\})$ & $>0$ & \\
\hline 59 & $-11-$ & $(0145678\{1\} 329\{5\})$ & $>0$ & \\
\hline$\overline{600}$ & $\overline{(014587\{P\} 6329)}$ & (0145876\{6\}329) & $\overline{0}$ & $13 \uplus_{3} 15.4$ \\
\hline 61 & $-11-$ & $(0\langle 4\rangle 145876\{2\} 329)$ & 0 & constructed in $[3,2.3]$ \\
\hline 62 & $-11-$ & $(0145876\{2\} 329\{4\})$ & $>0$ & \\
\hline 63 & $(014783\{P\} 2965)$ & 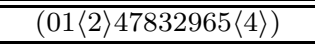 & 0 & constructed in [3, 2.3$]$ \\
\hline 64 & $-11-$ & $(01\langle 6\rangle 47832965)$ & 0 & constructed in $[3,2.3]$ \\
\hline 65 & $(07\langle P\rangle 89612345)$ & $(0\{3\} 7\{2\} 8961\{1\} 2345)$ & 0 & $4.2 \uplus_{1} 9.5$ \\
\hline 66 & $-11-$ & $(0\{1\} 78\langle 2\rangle 961\{3\} 2345)$ & 0 & $4.2 \uplus_{1} 9.5^{*}$ \\
\hline 67 & $-11-$ & $(0\langle 5\rangle 789612345\{1\})$ & 0 & \begin{tabular}{ll|l} 
restricted in [3] & $4.3] \mathrm{PH}$ \\
\end{tabular} \\
\hline 68 & $\begin{array}{ll}-11- \\
-1\end{array}$ & $(0\langle 1\rangle 789612345\{5\})$ & $>0$ & \\
\hline 69 & $-11-$ & $(0\langle 1\rangle 789\langle 4\rangle 612345\{1\})$ & $>0$ & \\
\hline$\overline{70}$ & $(07\langle P\rangle 89632145)$ & $(0,(0\{4\} 7\{2\} 89632145)$ & 0 & $8.2 \uplus_{1} 9.8$ \\
\hline 71 & $-11-$ & $(0\langle 2\rangle 7896\{2\} 321\{2\} 45)$ & 0 & $8.2 \uplus_{1} 9.8^{*}$ \\
\hline 72 & $\begin{array}{ll}-11- \\
-1\end{array}$ & $(07\{2\} 89\langle 4\rangle 632145)$ & $>0$ & \\
\hline 73 & 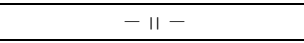 & $(0\{6\} 789632145)$ & $>0$ & \\
\hline$\overline{74}$ & $(0967\langle P\rangle 812345)$ & 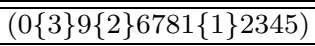 & 0 & $8.2 \uplus_{1} 9.2$ \\
\hline 75 & $-11-$ & $(096\langle 2\rangle 78\{1\} 1\{3\} 2345)$ & 0 & $8.2 \uplus_{1} 9.2^{*}$ \\
\hline 76 & 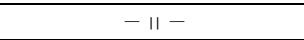 & $(096\langle 5\rangle 781\langle 1\rangle 2345)$ & 0 & $8.1 \uplus_{1} 9.8$ \\
\hline 77 & $-11-$ & $(096\langle 2\rangle 781\{3\} 234\{1\} 5)$ & 0 & $8.1 \uplus_{1} 9.8^{*}$ \\
\hline 78 & $-11-$ & $(096\langle 1\rangle 781\langle 5\rangle 2345))$ & $>0$ & \\
\hline 79 & $\begin{array}{ll}-11- \\
-1\end{array}$ & $(096\langle 1\rangle 78123\langle 5\rangle 45)$ & $>0$ & \\
\hline 80 & $-11-$ & $(09\langle 4\rangle 6\langle 1\rangle 781\langle 1\rangle 2345)$ & $>0$ & \\
\hline 81 & $-11-$ & $(09\langle 3\rangle 6\langle 2\rangle 781\{1\} 2345)$ & $>0$ & \\
\hline 82 & $\overline{-11-}$ & $(09\langle 4\rangle 6\langle 1\rangle 78123\langle 1\rangle 45)$ & $>0$ & \\
\hline 83 & $-11-$ & $(096\langle 5\rangle 78123\langle 1\rangle 45)$ & $>0$ & \\
\hline 84 & $\overline{(096783\{P\} 2145)}$ & 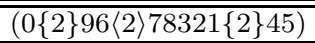 & 0 & $8.2 \uplus_{2} 11.2 \uplus_{2} 12.1$ \\
\hline 85 & $-11-$ & $(096783\langle 2\rangle 21\{4\} 45)$ & $>0$ & \\
\hline 86 & $-11-$ & $(096\langle 4\rangle 783\langle 2\rangle 2145)$ & $>0$ & \\
\hline 87 & $-11-$ & $(096783\langle 6\rangle 2145)$ & $>0$ & \\
\hline 88 & $(098123456\{P\} 7)$ & $(0\{1\} 9\langle 3\rangle 8\langle 2\rangle 1234567)$ & 0 & $8.1 \uplus_{1} 9.7^{*}$ \\
\hline 89 & $-11-$ & $(0\langle 1\rangle 9812345\{5\} 67)$ & 0 & $13 \uplus_{3} 15.1$ \\
\hline 90 & $-11-$ & $(09\langle 3\rangle 8\langle 2\rangle 1234\{1\} 567)$ & 0 & $8.2 \uplus_{1} 9.1^{*}$ \\
\hline 91 & $\overline{-11-}$ & $(09\langle 1\rangle 81234\{3\} 5\{2\} 67)$ & 0 & $8.2 \uplus_{1} 9.1$ \\
\hline 92 & $\overline{-11-}$ & $(098\langle 5\rangle 123\langle 1\rangle 4567)$ & 0 & $8.1 \uplus_{1} 9.7$ \\
\hline 93 & $-11-$ & $(0\langle 1\rangle 9\langle 4\rangle 812345\{1\} 67)$ & 0 & constructed in $[3,2.3]$ \\
\hline 94 & $-11-$ & $(09\langle 4\rangle 8\langle 1\rangle 1\langle 1\rangle 234567)$ & $>0$ & \\
\hline 95 & $\begin{array}{ll}-11- \\
-1\end{array}$ & $(09\langle 4\rangle 8\langle 1\rangle 123\langle 1\rangle 4567)$ & $>0$ & \\
\hline 96 & $-11-$ & $(098\langle 1\rangle 1\langle 5\rangle 234567)$ & $>0$ & \\
\hline 97 & $\overline{-11-}$ & $(098\langle 5\rangle 1\langle 1\rangle 234567)$ & $>0$ & \\
\hline 98 & $-11-$ & $(098\langle 1\rangle 123\langle 5\rangle 4567)$ & $>0$ & \\
\hline 99 & $-11-$ & $(0\langle 2\rangle 9\langle 3\rangle 81234\{1\} 567)$ & $>0$ & \\
\hline 100 & $-11-$ & $(0\langle 1\rangle 981234\{4\} 5\{1\} 67)$ & $>0$ & \\
\hline 101 & $-11-$ & $(0\langle 2\rangle 9\langle 3\rangle 8\{1\} 1234567)$ & $>0$ & \\
\hline 102 & $\begin{array}{ll}-11- \\
-1\end{array}$ & $(0\langle 5\rangle 9812345\{1\} 67)$ & $>0$ & \\
\hline
\end{tabular}




\begin{tabular}{|c|c|c|c|c|}
\hline No. & $\begin{array}{l}\mathbb{R} C_{1} \cup \mathbb{R} C_{1}^{\prime} \cup J \text { and } \\
\text { choice of pencil center }\end{array}$ & $\begin{array}{l}\mathbb{R} C_{1} \cup \mathbb{R} C_{1}^{\prime} \cup \mathbb{R} C_{5} \\
\text { (location of ovals) }\end{array}$ & $\bar{h}$ & Answer \\
\hline 103 & $(098143\{P\} 2567)$ & $(098\langle 2\rangle 1\{2\} 4\{2\} 32567)$ & 0 & $8.2 \uplus_{2} 11.4 \uplus_{2} 12.1$ \\
\hline 104 & $-\|-$ & $(098\langle 4\rangle 1\langle 2\rangle 432567)$ & $>0$ & \\
\hline 105 & $-11-$ & $(0981\{4\} 43\langle 2\rangle 2567)$ & $>0$ & \\
\hline 106 & $-11-$ & $(098143\langle 6\rangle 2567)$ & $>0$ & \\
\hline 107 & $-11-$ & $(09814325\{6\} 67)$ & $>0$ & \\
\hline 108 & $-11-$ & $(0981\{4\} 4325\{2\} 67)$ & $>0$ & \\
\hline 109 & $-11-$ & $(09814\{4\} 325\{2\} 67)$ & $>0$ & \\
\hline 110 & 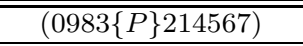 & $(098\langle 4\rangle 3\langle 2\rangle 214567)$ & 0 & $8.2 \uplus_{1} 9.7$ \\
\hline 111 & $-11-$ & $(0\{2\} 98\langle 2\rangle 321\{2\} 4567)$ & 0 & $8.1 \uplus_{1} 9.7^{*}$ \\
\hline 112 & $-11-$ & $(0983\langle 2\rangle 21\{4\} 4567)$ & $>0$ & \\
\hline 113 & $\overline{-11-}$ & $(0983\langle 6\rangle 214567)$ & $>0$ & \\
\hline 114 & $-11-$ & $(0\langle 2\rangle 98\{2\} 321\langle 2\rangle 4567)$ & $>0$ & \\
\hline$\overline{115}$ & $\overline{(0\{P, *\} 987612345)}$ & $\overline{(0\{4\} 9\{1\} 8761\{1\} 2345)}$ & 0 & $8.1 \uplus_{1} 9.2$ \\
\hline 116 & $-11-$ & $(0\{4\} 987\{1\} 61\{1\} 2345)$ & 0 & $8.1 \uplus_{1} 9.5$ \\
\hline 117 & $\overline{-11-}$ & $(0\{1\} 9876\langle 1\rangle 1\{4\} 2345)$ & 0 & $8.1 \uplus_{1} 9.2^{*}$ \\
\hline 118 & $\overline{-11-}$ & $(0\langle 1\rangle 98\{1\} 761\{4\} 2345)$ & 0 & $8.1 \uplus_{1} 9.5^{*}$ \\
\hline 119 & $\overline{-11-}$ & $(09876\langle 1\rangle 1234\{5\} 5)$ & $>0$ & \\
\hline 120 & $-11-$ & $(0\langle 1\rangle 98\{5\} 7612345)$ & $>0$ & \\
\hline 121 & $-11-$ & $(0\{5\} 9876\langle 1\rangle 12345)$ & $>0$ & \\
\hline 122 & $(098761\langle P, *\rangle 2345)$ & $(09\langle 1\rangle 8761\langle 5\rangle 2345)$ & $>0$ & \\
\hline 123 & $-11-$ & $(09\langle 5\rangle 8761\langle 1\rangle 2345)$ & $>0$ & \\
\hline 124 & $(09876123\langle P, *\rangle 45)$ & $(09\langle 1\rangle 876123\langle 5\rangle 45)$ & $>0$ & \\
\hline 125 & $-11-$ & $(09\langle 5\rangle 876123\langle 1\rangle 45)$ & $>0$ & \\
\hline 126 & $(0\langle P, *\rangle 987612345)$ & $(0\langle 4\rangle 98761\{1\} 2345\{1\})$ & 0 & $\begin{array}{llll}\text { restricted in [3] } & 0.1] & \mathrm{PH} \\
\end{array}$ \\
\hline 127 & $\begin{array}{c}(0987612345) \\
\text { (no necessary pencil) }\end{array}$ & $(09\langle 5\rangle 87612345\{1\})$ & - & $\begin{array}{l}\text { restricted by reduction } \\
\text { to } C_{2} \cup C_{5} \text { in } 3\end{array}$ \\
\hline 128 & $-11-$ & $(09\langle 1\rangle 87612345\{5\})$ & - & $-11-$ \\
\hline$\overline{\overline{129}}$ & $\overline{(2\langle P\rangle 109834567)}$ & 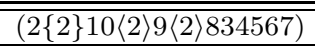 & $\overline{0}$ & 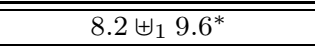 \\
\hline 130 & $-11-$ & $(21\langle 2\rangle 09834\{4\} 567)$ & 0 & $8.2 \uplus_{1} 9.6$ \\
\hline 131 & $-11-$ & $(2\{2\} 109\langle 2\rangle 8\langle 2\rangle 34567)$ & $>0$ & \\
\hline 132 & $-11-$ & $(21\langle 2\rangle 09\langle 4\rangle 834567)$ & $>0$ & \\
\hline 133 & $-11-$ & $(21\langle 6\rangle 09834567)$ & $>0$ & \\
\hline
\end{tabular}

lines in $L_{P}$ with that curve. If we unglue all circles via some standard perturbation, we obtain a link $K\left(C_{7}, P\right)$. Let $b\left(C_{7}, P\right)$ be a braid whose closure coincides with $K\left(C_{7}, P\right)$. For what follows, it is essential that the braid $b\left(C_{7}, P\right)$ is uniquely determined (up to conjugation in the group $B_{7}$ of braids of seven strings) by the mutual arrangement of the model of $C_{7}$ and the pencil $L_{p}$ in $\mathbb{R} P^{2}$.

It is known that the above braid must be quasipositive, which means that it can be written in the form $\prod_{j=1}^{k} \omega_{j} \sigma_{i_{j}} \omega_{j}^{-1}$, where $\sigma_{s}, s \in\{1,2, \ldots, 6\}$, are the standard generators of group $B_{7}$, and $\omega_{j}, j \in\{1,2, \ldots, k\}$, are some words written in the alphabet $\left\{\sigma_{1}, \ldots, \sigma_{6}, \sigma_{1}^{-1}, \ldots, \sigma_{6}^{-1}\right\}$. Now, suppose we are given a topological model of a conjectural curve $\mathbb{R} C_{7}$, together with any possible mutual location of the pencil $L_{P}$ and this model. If we find that the braid $b\left(C_{7}, P\right)$ is not quasipositive, then this model is not realizable by any algebraic curve of degree 7 . As a necessary condition of quasipositivity, we employ the Murasugi-Tristram inequality, which can be written in the form $h \leq 0$, where $h$ is a linear combination of the signature and defect of the link $K\left(C_{7}, P\right)$ and the algebraic length of the braid $b\left(C_{7}, p\right)$.

Almost all steps of the application of the Orevkov method (finding a word that determines the braid $b\left(C_{7}, P\right)$, computation of the invariant $h$, etc.) were realized as a computer algorithm in the paper [4] and were improved in [1, 8, 9. However, the choice of an appropriate point $P$ for the center of the pencil is done "manually". This choice is 
indicated in the second column of Table 1, where the meaning of the angle brackets or braces is the same as in rule 3 ) for codes in $\S 2$ above, and the star * means that we pick the point $P$ inside an oval in the respective region. After the position of $P$ is chosen, it is necessary to examine all possible locations of models with respect to the pencil $L_{P}$. As an example, we examine the arrangement coded by (0981432567).

In Figure 4.1, the dotted lines of the pencil $L_{p}$ are tangent lines that touch the odd branch of our quintic curve. The connected components of the complement to the model under study are denoted by Latin letters. These components must contain six ovals of the quintic curve, which lie outside one another. The admissible arrangements of ovals in the connected components have already been listed; for the example in question, see rows 103-109 of Table 1. In accordance with the Bézout theorem, the ovals can be located only in hatched areas of these components.

The mutual location of the odd branch $J$ and the lines $\mathbb{R} C_{1}$ and $\mathbb{R} C_{1}^{\prime}$ shown in Figure 42 is the same as in Figure 4.1. To turn Figure 4.1 into Figure 4,2, one should merely choose the line that intersects the model curve at the points 1-7 for the role of the boundary of the Poincaré disk. Finally, Figure 5 shows the affine part of the Poincaré disk of Figure 4,2 (the boundary of the Poincaré disk becomes the line at infinity, $P$ becomes the point at infinity of the ordinate axis, the pencil $L_{p}$ turns into the pencil of vertical parallel lines, and the hatched areas turn into hatched areas that must contain the six ovals).
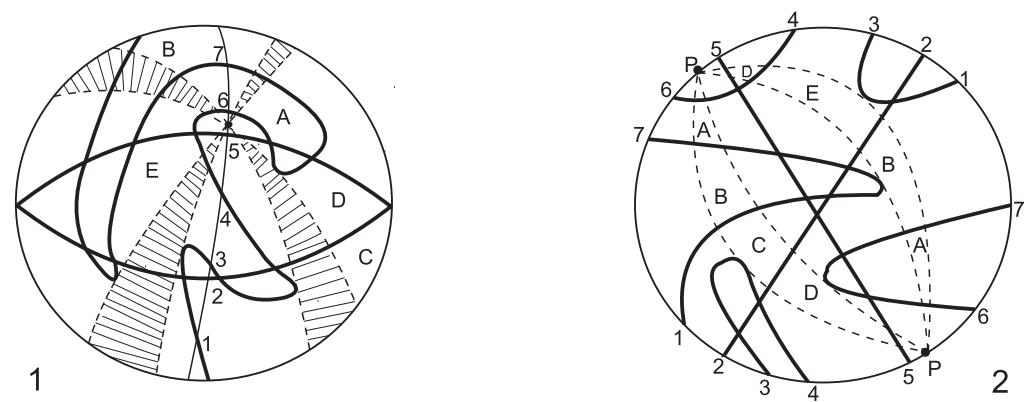

Figure 4

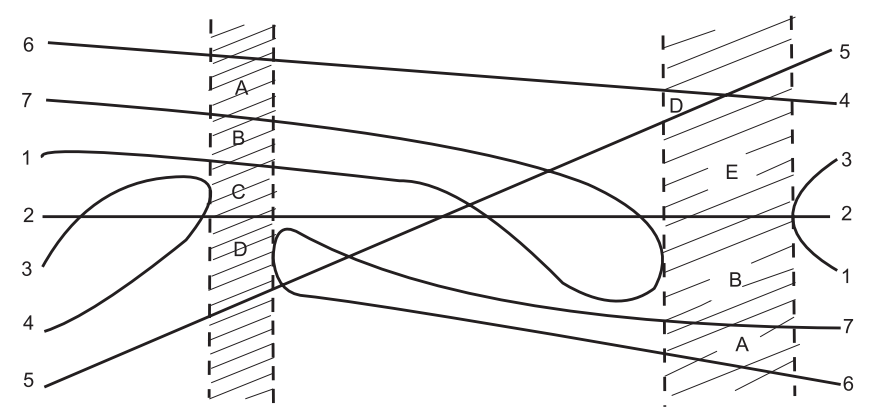

FiguRE 5

Next, using Figure 5, we compose a code, the parameters of which are the numbers of ovals in the horizontal strips of the hatched areas shown in Figure 5. With the help of a computer, we run through all collections of parameters corresponding to arrangements of ovals lying in the components $\mathrm{A}-\mathrm{E}$ and compute the invariant $h$ for each collection. If 
for every collection of parameters we obtain $h>0$ (we mark this in the fourth column of Table 1), then no location with the given arrangement of ovals in the components A-E is realizable by an algebraic curve of degree 7 .

\section{$\S 4$. Construction by the Viro method}

In this section we construct curves of degree 5 having some set of singular points and then smooth them by the Viro method.

\subsection{Curves with singularities of types $D_{4}$ and $A_{4}$.}

Lemma 1. There exists a curve of degree 5 with singularity $D_{4}$ at the point $(0: 0: 1)$ and $A_{4}$ at $(1: 0: 0)$ shown in Figure 6.1. This curve has the Newton polygon with vertices $(0,3),(0,5),(3,0)$ and the chart shown in Figure 6.2.
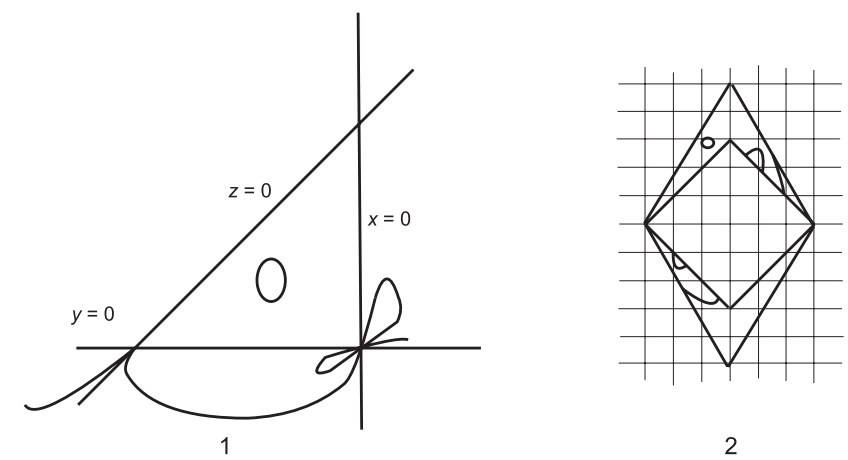

2

FiguRE 6

Proof. Applying the hyperbolism $(x: y: z) \mapsto\left(x y: y z: z^{2}\right)$ to the curve of degree 3 shown in Figure 7, we get the required curve.

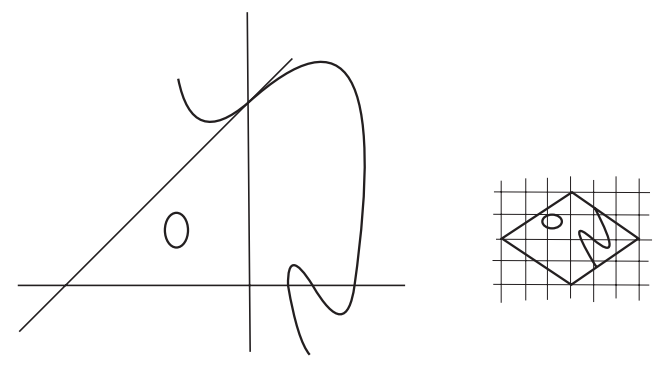

FigURE 7

The following lemma provides smoothing of the singularities of type $D_{4}$. It was proved in [7] (for the details, see also Lemmas 1.1-1.4 in [8]).

Lemma 2. For any numbers $0<a_{1}<a_{2}<a_{3}$, there exist two curves of degree 3 passing through the points $\left(1: a_{i}: 0\right), i \in\{1,2,3\}$ and having the charts shown in Figure 8 .

The following lemma provides smoothing of the singularities of type $A_{4}$.

Lemma 3. There exist nine curves of degree 5 having the Newton polygon with vertices $(0,5),(5,0),(3,0)$ and the charts shown in Figure 9 . 


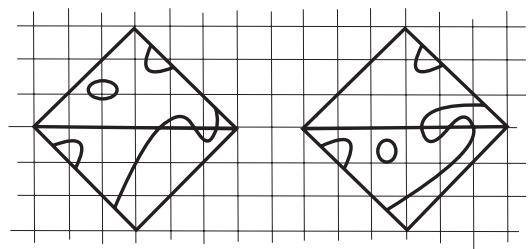

Figure 8

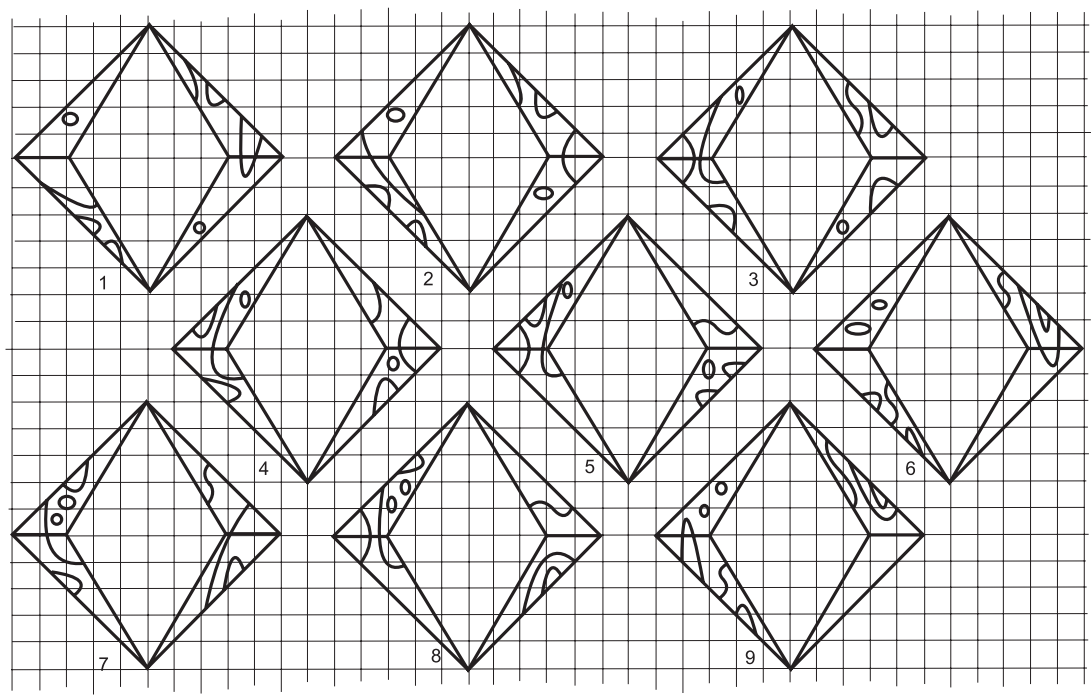

FIGURE 9

Proof. Three curves of degree 5 in Figure 10] were constructed in [9] (their codes in [9] are $\left.f_{5}^{100}, f_{5}^{110}, f_{5}^{010}\right)$. Each of them has two ovals, an odd branch, and a singularity of type $E_{8}$ at the point $(0: 0: 1)$. If we choose coordinate systems as shown in Figure 10, i.e., the axes $x=0$ and $z=0$ are the same and the axis $y=0$ takes positions $1-9$ consecutively, then we obtain curves with the charts depicted in Figures 9.1-9, respectively.

Theorem 4.1. The 35 topological models that have symbol $\uplus_{1}$ in the fifth column of Table 1 are realizable by unions of a quintic curve and a pair of lines.

Proof. Consider the triples of curves provided by Lemmas 1-3. The first element of every triple is a curve of degree 5 with the chart shown in Figure 6. The second element is a curve with one of the two charts shown in Figure 8. The third element is either a curve with one of the nine charts shown in Figure 9] or a curve with one of the nine new charts obtained from the charts shown in Figure 9 by the transformation $(x, y) \mapsto(-x,-y)$. Every triple satisfies the Viro patchworking theorem proved in [5. As a result of every such patchworking, we obtain an arrangement of $\mathbb{R} C_{5} \cup \mathbb{R} C_{1} \cup \mathbb{R} C_{1}^{\prime}$ that realizes the quadruple (1.1) and satisfies conditions (i)-(iv), where the axes $y=0$ and $z=0$ play the roles of the lines $\mathbb{R} C_{1}$ and $\mathbb{R} C_{1}^{\prime}$. For example, the chart of a curve that realizes model 2 of Table 1 can be obtained by patchworking the charts shown in Figures 6, 2, 8, 1, and 9,9. In the last column of Table 1, we indicate the number of charts in Figures 8 and 9 that are involved in patchworking. The star means that a chart in Figure 9,9 was transformed by the rule $(x, y) \mapsto(-x,-y)$. 

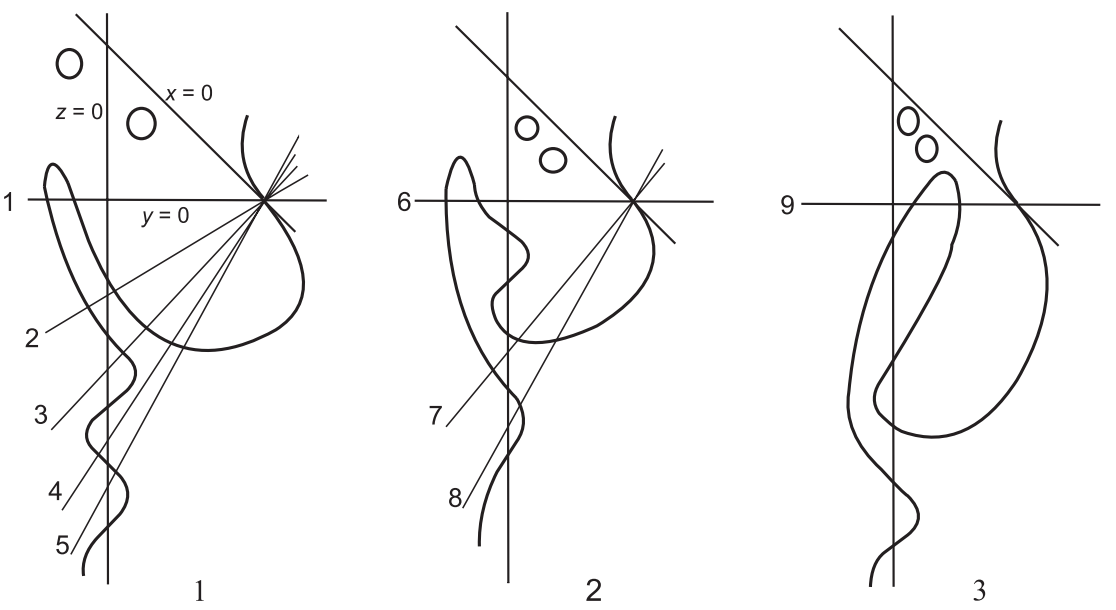

FIGURE 10

Remark. The number of distinct triples of charts considered in the proof equals $1 \times 2 \times$ $(9+9)=36$, but the patchworkings $8.1 \uplus_{1} 9.1^{*}$ and $8.1 \uplus_{1} 9.3^{*}$ give the same model, so that we get 35 instead of 36 topological models (1.1).

\subsection{Curves with singularities of types $D_{4}$ and $A_{3}$.}

Lemma 4. There exist two curves of degree 5, shown in Figures 11.1 and 11], having the Newton polygon with the vertices $(0,3),(4,1),(5,0),(3,0)$, and having the charts shown in Figures 11,2 and 11,4, respectively.
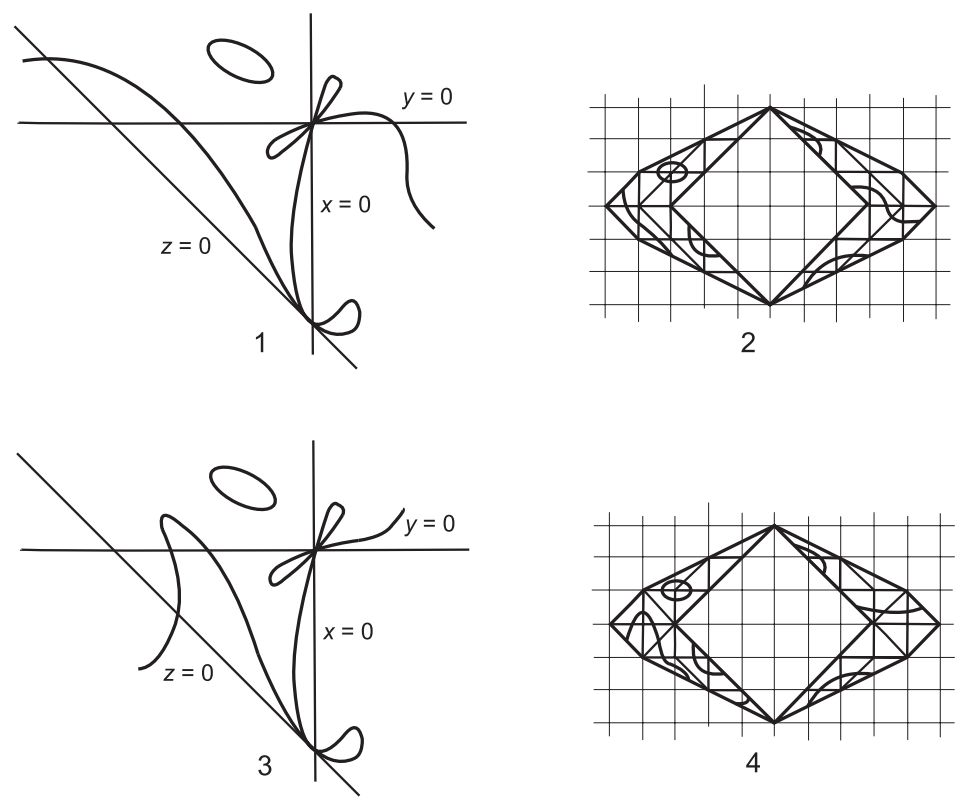

FIGURE 11

Proof. Patchworking of these curves is shown in Figures 11,2 and 11,4. 
Lemma 5. There exist two curves of degree 5 having the Newton polygon with vertices $(0,3),(0,5),(4,1)$ and having the charts shown in Figures 12,1 and 12,2 , respectively.

Proof. These curves are also obtained by patchworking; see Figure 12 .

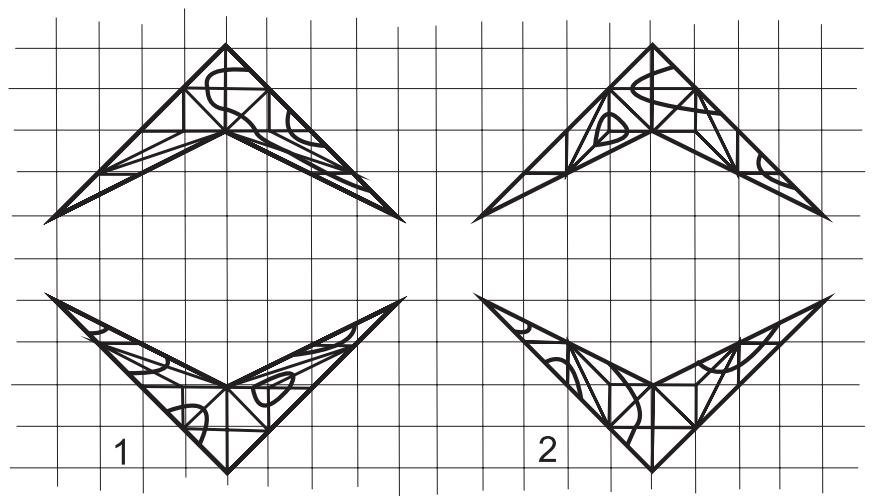

FiguRE 12

Theorem 4.2. The two topological models shown in rows 84 and 103 are realizable by unions of a quintic curve and a pair of lines.

Proof. We consider triples provided by Lemmas 2, 4, 5 and patchwork them as is shown in the fifth column of rows 84 and 103 of Table 1 . The axes $y=0$ and $z=0$ play the role of the lines $\mathbb{R} C_{1}$ and $\mathbb{R} C_{1}^{\prime}$.

\subsection{Curves with singularity of type $A_{3}$.}

Lemma 6. There exists a curve of degree 5 having the Newton polygon with the vertices $(0,2),(0,5),(5,0)$ and the chart shown in Figure 13 .

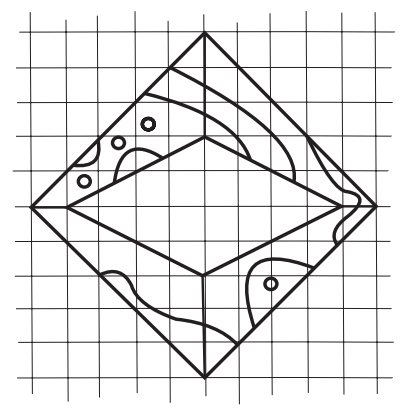

FiguRE 13

Proof. Consider the arrangement of a conic $K$ and two lines $L$ and $y=0$ shown in Figure 14, 1 (the axis $y=0$ is tangent to $K$ at the origin $O$ ). Let $K$ and $L$ have equations $C_{2}=0$ and $L=0$, respectively.

Let four points $a_{1}, a_{2}, a_{3}, a_{4} \in K$ be close to $O$. Let $L_{1}=0, L_{2}=0, L_{3}=0$ be equations of the lines $a_{1} O, a_{2} O, a_{3} a_{4}$, respectively. Consider the curve of degree 3 with the equation $C_{3} \equiv C_{2} L+t L_{1} L_{2} L_{3}=0$. If $t$ is a small parameter $(|t| \ll 1)$ with a 

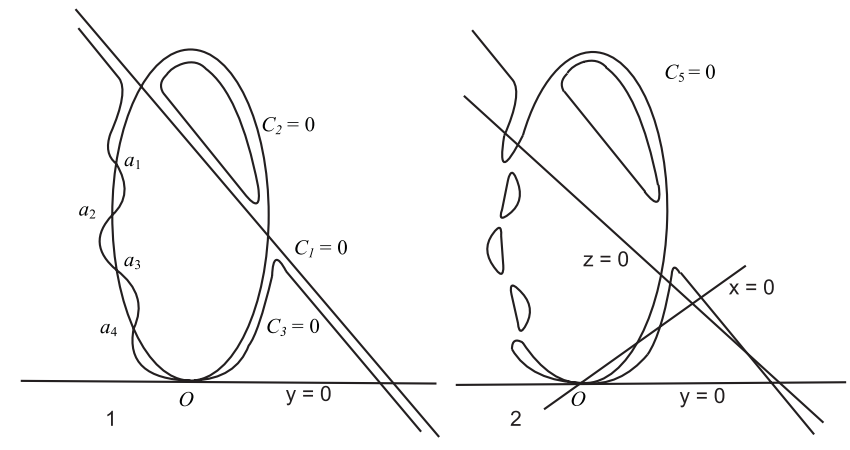

FiguRe 14

suitable sign, then this curve looks like that shown in Figure 14,1. Let $C_{5}$ denote the result of smoothing the union of the conic $K$ and the cubic curve $C_{3}$ such that the double points at $a_{1}, a_{2}, a_{3}, a_{4}$ are smoothed as shown in Figure 14, 2 , but the $A_{3}$-singularity at $O$ remains intact. Choose a projective coordinate system with axes $x=0, y=0, x=0$, as shown in Figure 14,2. In this coordinate system, the chart of the curve $C_{5}$ is as shown in Figure 13.

Lemma 7. There exist four curves of degree 4 having the Newton polygon with vertices $(0,0),(0,2)$, and $(4,0)$ and having the charts shown in Figure 15.

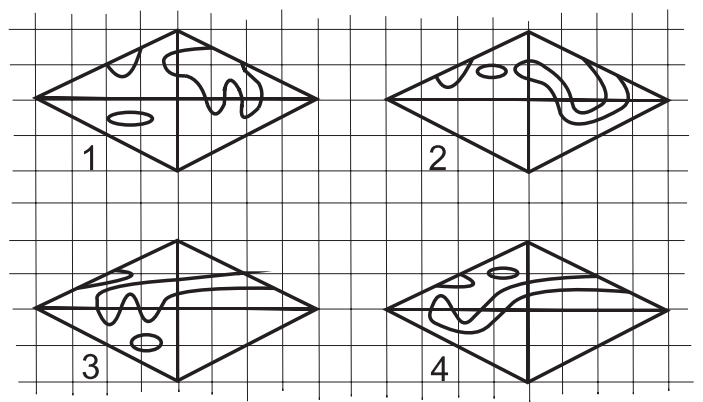

FIGURE 15

Proof. Such curves are well known. In particular, in [9], one can find patchworking for the charts of Figures [15] 1 and 15,2 (in 9] they have codes $f^{000}$ and $f^{010}$, respectively). The charts shown in Figures 15],3 and 15.4 can easily be obtained from the charts of Figures 15, 1 and 15,2: the axis $x=0$ can be rotated suitably around the point $(0: 1: 0)$.

Theorem 4.3. The five topological models marked with $\uplus_{1}$ in the fifth column of Table 1 ( see rows $7,37,55,60,89$ ) are realizable by unions of a quintic curve and a pair of lines.

Proof. Since the curves provided by Lemmas 2 and 7 satisfy the Viro patchworking theorem [5], we can patchwork the chart of Figure 8 in turn with the charts of Figures 15, 1-15.4. As a result, we get charts of curves that realize the required models. For the models of rows 55,60 , and 89 , the axes $x=0$ and $y=0$ play the role of the lines 
$\mathbb{R} C_{1}$ and $\mathbb{R} C_{1}^{\prime}$, and for rows 7 and 37 , the same role is played by the axes $y=0$ and $z=0$.

5. Completion of the classification by S. Yu. Orevkov in [3. We studied 114 models of Table 1 after which the realizability question remained open for 18 models. We communicated this information to S. Yu. Orevkov, who studied a similar problem, and he completed the classification. In the paper [3, he proved that the 9 models of rows 21 , $22,27,28,56,61,63,64,93$ of Table 1 are realized by algebraic curves of degree 7 , and the 9 models of rows $16,41,48,49,57,67,126,127,126$ are nonrealizable. Note that for the models of rows 41 and 126 the answer was announced in [3] without proof.

Remark. Also in [3], Orevkov enlarged the list of examples (which he obtained in earlier papers) of topological models that are nonrealizable by algebraic curves of the degree in question, but are realizable by pseudoholomorphic curves. In particular, in [3, it was proved that models $16,41,57,67,126$ of Table 1 are realizable by pseudoholomorphic curves (we mark them by the symbol "PH" in the fifth column).

\section{REFERENCES}

[1] A. B. Korchagin and G. M. Polotovskii, On arrangements of a plane real quintic curve with respect to a pair of lines, Commun. Contemp. Math. 5 (2003), no. 1, 1-24. MR.1958018 (2004a:14060)

[2] G. M. Polotovskiur, A catalogue of $M$-splitting curves of order six, Dokl. Akad. Nauk SSSR 236 (1977), no. 3, 548-551; English transl. in Soviet Math. Dokl. 18 (1977). MR0460339 (57:333)

[3] S. Yu. Orevkov, Arrangements of an $M$-quintic with respect to a conic that maximally intersects its odd branch, Algebra i Analiz 19 (2007), no. 4, 174-242; English transl., St. Petersburg Math. J. 19 (2008), no. 4, 625-674. MR2381938(2009d:14076)

[4] _ Link theory and oval arrangements of real algebraic curves, Topology 38 (1999), no. 4, 779-810. MR 1679799 (2000b:14066)

[5] O. Ya. Viro, Gluing of algebraic hypersurfaces, elimination of singularities, and constructions of curves, Proceedings of the International Topological Conference (Leningrad, 1982), Nauka, Leningrad, 1983, pp. 149-197. (Russian)

[6] _ Gluing of plane real algebraic curves and constructions of curves of degrees 6 and 7, Topology (Leningrad, 1982), Lecture Notes in Math., vol. 1060, Springer, Berlin, 1984, pp. 187-200. MR:0770238 (87i:14029)

[7] _ Real algebraic manifolds with prescribed topological properties, Doctor's Diss., Leningrad. Gos. Univ., Leningrad, 1983. (Russian)

[8] A. B. Korchagin, Smoothing of 6-fold singular points and constructions of the 9th degree M-curves, Topology of Real Algebraic Varieties and Related Topics, Amer. Math. Soc. Transl. Ser. 2, vol. 173, Amer. Math. Soc., Providence, RI, 1996, pp. 141-155. MR.1384314 (97f:14058)

[9] A. B. Korchagin and D. E. Smith, Patchworking singularities $A_{\mu}$ and $D_{\mu}$ and meanders of their smoothing, Zap. Nauchn. Sem. S.-Peterburg. Otdel. Mat. Inst. Steklov. (POMI) 299 (2003), 193-217; English transl., J. Math. Sci. (New York) 131 (2005), no. 1, 5366-5380. MR2038262 (2004k:14110)

Lobachevsky University, 23 Gagarin Ave., Nizhny Novgorod 603950, Russia

E-mail address: korchagin@rf.unn.ru

Lobachevsky University, 23 Gagarin Ave., Nizhny Novgorod 603950, Russia

E-mail address: polot@uic.nnov.ru

Received 24/DEC/2007

Translated by A. B. KORCHAGIN 